\title{
Directing attention to movement effects enhances learning: A review
}

\author{
GABRIELE WULF \\ University of Nevada, Las Vegas, Nevada \\ and \\ WOLFGANG PRINZ \\ Max Planck Institute for Psychological Research, Munich, Germany
}

\begin{abstract}
Studies investigating the influence of the learner's focus of attention, induced by instructions or feedback, on motor skill learning are reviewed. In general, directing performers' attention to the effects of their movements (external focus of attention) appears to be more beneficial than directing their attention to their own movements (internal focus of attention). Preliminary evidence is presented indicating that an internal attentional focus constrains the motor system by interfering with natural control processes, whereas an external focus seems to allow automatic control processes to regulate the movements. Support for the view that actions are controlled by their anticipated effects comes from research demonstrating functional variability in motor control, as well as the benefits of purposeful activity in occupational therapy. We explain these results in terms of the ideomotor principle of human actions (James, 1890) and its more modern derivatives (Hommel, 1996; Prinz, 1990, 1997).
\end{abstract}

It has long been recognized that being too concerned with, or even just paying attention to, one's movements can disrupt the performance of well-practiced skills (Bliss, 1892-1893; Boder, 1935). For example, Walter Schneider shares a personal experience that he had in downhill skiing: When he found himself thinking about which foot was carrying his weight in a turn, he noticed "substantial performance decrements (i.e., many falls) for the remainder of the slope" (Schneider \& Fisk, 1983, p. 133). How one can take advantage of the detrimental effects of self-focused attention is pointed out by Gallwey $(1982$, p. 8). He suggests that you ask your tennis opponent, while switching courts, what he is doing that is making his forehand so good today, since this should make him think about his swing and disrupt his performance. Not only is there plenty of anecdotal evidence for this phenomenon (e.g., Kimble \& Perlmuter, 1970; Klatzky, 1984; Masters, 1992; Schmidt, 1988), but more recently, the detrimental effects of directing attention to one's movement coordination have also been demonstrated experimentally. Baumeister (1984) found that increased conscious attention directed to one's own performance, induced, for example, by social pressure to perform well, disrupted the performance of a wellpracticed video game. When customers at a video game

This study was supported by Grant Pr 188/18-2 from the Deutsche Forschungsgemeinschaft. The authors thank Richard A. Schmidt and two anonymous reviewers for their valuable comments on an earlier version of the manuscript. Correspondence concerning this article should be addressed to G. Wulf, Department of Kinesiology, University of Nevada, Las Vegas, 4505 Maryland Parkway, Las Vegas, NV 891543034 (e-mail: gabriele.wulf@ccmail.nevada.edu) arcade were asked to try to get the best score they could, there was a significant drop in performance (with an average of $25 \%$ ), as compared with the previous trial, which had been observed by the experimenter without the participant's awareness. Also, Wulf and Weigelt (1997, Experiment 2), using a ski simulator task, found that when participants were given instructions regarding the most effective movement technique after several days of practice, significant performance decrements occurred-presumably, because of the increased attention participants directed to their performance.

Perhaps even more important, there is also evidence to suggest that the learning of motor skills can be degraded if the learner pays too much attention to his or her performance. For example, Wulf and Weigelt (1997, Experiment 1 ) found that providing participants with instructions about how to best perform the ski simulator task also hampered learning in beginners. That is, not only did these instructions not enhance learning, as compared with no instructions, but they even degraded learning. These findings are quite worrisome if one considers that the instructions and feedback provided to learners in an attempt to guide them to the correct movement form-for example, in sport or physical therapy settings-typically refer to the spatiotemporal coordination of various movement components. Thus, the instructions almost necessarily direct the learner's attention to his or her performance. On the other hand, these findings suggest that there is a potential for optimizing the instructions or feedback provided in training to enhance the learning process.

In this paper, we review recent studies directed at investigating how the effectiveness of instructions and feedback 
can be enhanced to facilitate the learning of motor skills. As we will show, providing instructions and feedback that direct the performer's attention to the effects of his or her movements generally seems to be more beneficial than directing the learners' attention to their own movements. In addition to summarizing findings related to the differential effectiveness of different attentional foci, we also review preliminary evidence for different motor control processes associated with these foci of attention that could be responsible for the observed differences in performance and learning. Furthermore, we discuss theoretical principles that can account for these findings, including the common-coding account advanced by Prinz $(1990,1997)$. Finally, related findings from motor control and occupational therapy research will be presented, which also seem to indicate that actions are usually controlled by their anticipated effects.

\section{INSTRUCTIONS AND ATTENTIONAL FOCUS}

\section{Differential Learning Effects of Instructions Inducing an Internal Versus External Focus of Attention}

In a recent series of studies, Wulf and colleagues (e.g., Wulf, Höß, \& Prinz, 1998; Wulf, Lauterbach, \& Toole, 1999; Wulf, McNevin, \& Shea, 2001; Wulf, Shea, \& Park, in press) investigated how the effectiveness of instructions could be enhanced by manipulating the learner's focus of attention induced by the instructions. In these studies, it was consistently demonstrated that instructions that directed the learners' attention to the effects of their movements on the environment (e.g., the implement or apparatus) - that is, that induced an external focus of attention-are more effective than instructions directing their attention to the movements themselves (i.e., that induced an internal attentional focus). In the first study showing learning advantages of an external focus of attention, Wulf et al. (1998, Experiment 1) manipulated the attentional focus of participants attempting to learn slalom-type movements on a ski simulator. Whereas one group of performers was instructed to focus on the force exerted by their feet (internal focus), another group was instructed to focus on the force exerted on the wheels of the platform, which were directly under their feet (external focus). In addition, there was a control group without additional instructions. The external-focus group that focused on the effects of their movements on the apparatus demonstrated more effective learning than did both the internal-focus group and the control group. In fact, the internal-focus instructions were no more effective than no instructions at all (control group). ${ }^{1}$

The learning advantages of an external attentional focus were replicated in a second experiment (Wulf et al., 1998, Experiment 2), in which participants were required to learn to balance on a stabilometer. One group of participants was instructed to focus on keeping their feet horizontal (internal focus), whereas another group was instructed to focus on keeping two markers attached to the stabilometer platform (external focus) directly in front of their feet horizontal. Thus, the actual locus of attention differed only minimally between groups. Yet, in the former case attention was directed toward the performer's body movements, whereas in the latter case it was directed toward the effects of the performer's actions on the platform. Despite the minimal difference in instructions, on a retention test performed 1 day after the end of practice, the external-focus group showed superior balance learning, relative to the internal-focus group.

A study by Wulf et al. (1999) attempted to determine the generalizability of the external focus advantages to the learning of a sport skill under field-like conditions. In their study, participants without experience in golf practiced pitch shots. In this case, one group received instructions, like those typically given by golf instructors, to focus on the swing of their arms (internal focus). Another group was instructed to focus on the pendulum-like motion of the club (external focus). The external focus instructions greatly enhanced the accuracy of the shots not only in practice, but also in a retention test, relative to internal focus instructions. This study was the first demonstration of the generalizability of the advantages of focusing on the movement effects, rather than on the movements themselves, to the acquisition of more real-world skills.

More recently, Maddox, Wulf, and Wright (1999) also found benefits of external focus instructions for the learning of a tennis skill (backhand stroke cross-court). Learners instructed to focus on the trajectory of the ball and its landing point demonstrated a greater accuracy in their shots than did learners instructed to focus on their backswing and the racket-ball contact point-that is, their movement technique. This was seen not only during practice, but also in retention, as well as in a transfer test, in which participants were required to hit the ball to the other side of the backcourt. Importantly, Maddox et al. (Experiment 2) also showed that these learning advantages in the movement outcome were not achieved at the expense of the movement form. Expert rating revealed that the internal and the external focus groups were similar in the quality of their movement technique.

To examine whether there are individual differences in the preference for, and perhaps in the effectiveness of, the attentional focus or whether the advantage of an external attentional focus is a general phenomenon, Wulf, Shea, and Park (in press) gave participants the option to adopt either an internal or an external focus of attention. Participants learning to balance on the stabilometer were asked to find out for themselves which type of attentional focus seemed to be more effective. The results showed that more participants chose to focus on markers attached to the board in front of their feet (external focus) than on the feet (internal focus). Moreover, participants who adopted an external focus of attention demonstrated superior balance performance in retention than did participants with an internal focus. These results suggest that individual differences do not play a significant role in the relative effec- 
tiveness of an external versus internal focus of attention. Rather, the benefits of an external focus appear to be more general in nature.

Overall, these studies (Maddox et al., 1999; Wulf et al., 1998; Wulf et al., 1999; Wulf, Shea, \& Park, in press) provide consistent evidence that instructions that are phrased in such a way as to induce an external attentional focus by directing the performers' attention to the effects of their movements can enhance the learning processes, compared with instructions that direct performers' attention to their own movements. In principle, this effect could be the movement of an implement (e.g., golf club, tool) manipulated by the performer but also, for example, the trajectory of an object being hit or thrown (but see The Influence of the Distance of the External Effect and Theoretical Accounts sections for qualifications). The results of a recent study by Riley, Stoffregen, Grocki, and Turvey (1999) are nicely in line with the findings reported above. Riley et al. measured postural sway when participants, standing upright with their eyes closed, touched a curtain very lightly with their fingertips. A curtain was used because it would not provide any mechanical support for posture. Interestingly, touching the curtain significantly reduced postural fluctuation, as compared with not touching it—but only when the participants were asked to minimize movements of the curtain resulting from their touch (touch-relevant condition). When the participants were told that touching the curtain was irrelevant for the experiment (touch-irrelevant condition), postural sway was basically the same as under notouch conditions. These findings indicate that it was not simply the sensory information derived from the touch that reduced postural sway (see Jeka, Schöner, Dijkstra, Ribeiro, \& Lackner, 1997, for the influence of somatosensory information on postural control). Rather, the addition of a suprapostural goal (i.e., keeping the curtain still) resulted in spontaneous reductions in postural fluctuations to facilitate the achievement of this goal. These findings corroborate the view that instructing individuals to focus on an external movement effect enhances their performance by allowing performance to be mediated by automatic control processes (see the Attentional Focus and Motor Control section). The question remains, however, whether it is essential to focus on the movement effect or whether it is sufficient not to focus on the movements themselves. This question will be addressed in the next section.

\section{Focusing on the Movement Effect or Not Focusing on the Movements?}

On the basis of anecdotal evidence that suggests that expert performers do not seem to concentrate on their movement pattern when performing a highly practiced skill, but rather perform the skill automatically (e.g., Gallwey, 1982; Garfield \& Bennett, 1985), Singer (e.g., Singer, 1985, 1988; Singer \& Suwanthada, 1986) argued that instructing learners to be consciously aware of their body movements during the execution of a skill might not be very effective. Because attempting to perform a movement skill as if it were automatic did not appear to be tenable for beginners either, Singer $(1985,1988)$ developed his five-step approach as a compromise between awareness and nonawareness strategies. The five steps include (1) readying, (i.e., attaining an optimal emotional state, thinking positively), (2) imaging, (i.e., going through the motion mentally, "feeling" the movement), (3) focusing, (i.e., concentrating on one relevant cue and thinking only of this cue to block out all other thoughts), (4) executing the movement, while not thinking about the act itself or the possible outcome, and (5) evaluating, (i.e., assessing the outcome and planning adjustments for the next trial, if time permits; e.g., Singer, 1988).

Thus, this approach contains, as an important third step (focusing), the "simulation" of the attentional strategies presumably used by experts. Learners are instructed to concentrate on one relevant cue, such as the seams of a tennis ball or the dimples of a golf ball, to prevent the performer from focusing on what he or she is doing during movement execution (e.g., Singer, 1988; Singer et al., 1991; Singer, Lidor, \& Cauraugh, 1993). That is, a critical assumption underlying the five-step approach is that performers should not "think of anything about the act itself or the possible outcome" (Singer, 1988, p. 56). The effectiveness of the five-step approach relative to awareness strategies (where participants are instructed to think about their performance and to be aware of their movements), nonawareness strategies (where participants are instructed only to focus on a relevant cue), and control conditions without attentional focus instructions has been shown in a number of studies (Lidor, Tennant, \& Singer, 1996; Singer, Lidor, \& Cauraugh, 1993, 1994).

These findings are in line with those of Wulf et al. (e.g., Maddox et al., 1999; Wulf et al., 1998; Wulf et al., 1999; Wulf, Shea, \& Park, in press) in demonstrating the detrimental effects of self-focused attention, relative to attention directed toward an external cue. However, contrary to the approach promoted by Singer $(1985,1998)$, where external cues (e.g., ball, target) are used to prevent performers from attending to their movements, in the Wulf et al. studies the external cues were related to the effects of the performer's movements (e.g., force exerted on the wheels of the ski simulator platform, movements of the markers on the stabilometer platform, swing of the golf club).

Therefore, the purpose of a study by Wulf, McNevin, Fuchs, Ritter, and Toole (2000, Experiment 1) was to examine whether it is indeed critical and more advantageous to focus on the movement effects, as was suggested by the Wulf et al. studies, or whether it is sufficient not to focus on one's own movements (and to focus on some other cue instead), as was implied by Singer $(1985,1988)$. Specifically, the experiment was designed to determine whether there are differences in the effectiveness of instructions that direct the learners' attention to the effects of their movements, as compared with directing attention to an external cue that is not related to the movement effect. To examine this question, participants without experience in 
tennis were taught the forehand stroke and practiced hitting tennis balls to a target on the other side of the court. Whereas one group was instructed to focus on the ball approaching them (antecedent), another group was instructed to focus on the ball leaving the racket (effect). That is, the participants in both groups adopted an external focus of attention by focusing on the ball, but only the participants in the latter group directed their attention to the movement effect. If the benefits of an external focus of attention were simply due to the fact that they prevent performers from attending to their own movements, no group differences would be expected. If, however, focusing on the movement effect is more beneficial for learning, those participants focusing on the effects of their actions should demonstrate superior retention performance, relative to the participants focusing on the antecedents of their actions. The results showed that the group instructed to focus on the effect of their movements (arc of the ball they hit) demonstrated better retention performance than did the group instructed to focus on the antecedents (approaching ball). This suggests that the critical issue was not the external focus per se but whether attention was directed to the action effect.

Of course, actions often have more than one effect. For example, when hitting a golf ball, the performer's movements not only have an effect on the motion of the club, but also determine the trajectory and landing point of the ball. Thus, one might ask which of those effects the performer should focus on (or whether this matters at all). It is possible, for example, that focusing on a more distant effect is more advantageous than focusing on an effect that is closer to the body, because in the former case the effect might be easier to distinguish from the body movements that produced the effect. It is also conceivable-especially in the case of complex movement patterns- that focusing on an effect that is related to the movement technique (e.g., club motion in golf) might be more beneficial than focusing on a more distant but less technique-related effect (e.g., trajectory of the golf ball). A few studies have begun to address these issues and will be discussed next.

\section{The Influence of the Distance of the External Effect}

A comparison across the experiments by Wulf and colleagues (Maddox et al., 1999; Wulf et al., 1998; Wulf et al., 1999) seemed to indicate that the advantages of an external focus were enhanced (and found to occur earlier in the learning process) as the distance of the external effect from the body increased. For example, in the golf study by Wulf et al. (1999), where the distance between the arms and the clubhead was relatively large, the advantage of the external focus was found almost immediately in acquisition and remained present throughout the retention test. This was also true for the tennis task used by Maddox et al. (1999), where the external-focus participants directed their attention to the trajectory of the ball. On the ski simulator task (Wulf et al., 1998, Experiment 1), where the external cues (wheels) were located under the feet, the external- focus benefits were seen only at the end of the first day of practice and remained present throughout the second day of practice, as well as during the retention test on Day 3. Finally, for the balance task (Wulf et al., 1998, Experiment 2), where the distance between the feet and the markers on the platform was very small (the feet touched the markers), the advantage of the external focus became apparent only during the retention test after 2 days of practice. Thus, by increasing the distance of the movement effects from the body, the advantages of an external, relative to an internal, focus of attention seemed to be more pronounced.

On the basis of this observation, McNevin, Shea, and Wulf (2001) speculated that a greater distance between the body and the remote effect produced by its movements might further enhance the learning advantage associated with an external focus of attention. They argued that effects that occur in close spatial proximity to the body might be less easily distinguishable from the body movements than are more remote effects. Thus, directing attention to proximal effects should produce results more similar to those found for an internal focus of attention condition. To test this hypothesis, McNevin et al. (2001) manipulated the distance of the external movement effects that the performer's attention was directed to by having different groups practicing the stabilometer task focus on markers located at different distances from the body (feet). In one condition, performers were asked to focus attention on markers right in front of their feet (similar to Wulf et al., 1998, Experiment 2). In the other two conditions, performers were instructed to focus their attention on markers that were placed further to the outside or inside of the platform, respectively (both approximately $20 \mathrm{~cm}$ away from the feet). Markers on both the outside and the inside were used to ensure that any learning benefits associated with the distance manipulation could not be attributed to the different amounts of displacement of the different markers as a function of their distance from the axis of rotation. (The further the markers are placed to the outside of the platform, the greater their displacement.) If the distance of the attentional focus from the body (feet) is critical for the effectiveness of an external focus, focusing on the far markers-independent of whether they are on the outside or the inside of the platform-should result in learning benefits, as compared with focusing on the markers close to the feet. The results indeed showed that participants focusing on either of the far markers demonstrated better performance on a retention test than did participants focusing on the near markers. Also, the two groups that concentrated on the far markers showed very similar performances. Thus, the study by McNevin et al. (2001) provided preliminary evidence for the assumption that focusing on effects that occur at a greater distance from the body might be more effective because they are more easily distinguishable from the body than are close effects.

These findings were recently replicated and extended in a study by Park, Shea, McNevin, and Wulf (2000). In this experiment, the distance of the attentional focus was in- 
creased even further by having participants focus on markers located at the end of two sticks that were attached to the stabilometer platform in front of their feet, but at a distance of about $1 \mathrm{~m}$. This group of participants demonstrated even better balance learning than did groups that practiced under the same conditions as the far and near groups in the McNevin et al. (2001) study, thus providing additional evidence for the benefits of a more distant attentional focus.

These findings raise the question of whether the effectiveness of the attentional focus generally increases as its distance increases or whether there is an optimal distance. A study by Wulf et al. (2000, Experiment 2) suggests that focusing on more remote effects is not always more beneficial. In their study, the participants' task was to hit golf balls to a target. Whereas one group was instructed to focus on the swing of the club, the attention of the other group was directed to the anticipated trajectory of the ball and the target. That is, the participants of the latter group focused on a more distant effect than did those of the former group. The results showed that, as compared with instructing participants to focus on the ball trajectory and target, instructing them to focus on the club motion actually resulted in a greater accuracy of their shots, not only in practice, but also in a retention test.

Thus, it is possible that not only very small distances, but also relatively large distances of the effect that the performer focuses on are not optimal for learning. Rather, focusing on an effect at an "intermediate" distance might be most beneficial. Whereas close effects seem to be less advantageous because they are difficult to distinguish from the body movements that produced them, distant effects might have the disadvantage that they are too difficult to relate to those movements. Especially for novices who typically need to develop an image of the goal movement pattern-in particular, when it comes to complex skills-focusing on an effect that is not directly related to the movement technique might not be very effective. In the golf study by Wulf et al. (2000, Experiment 2), for example, the same ball trajectory could have been produced with different club motions (and different degrees of efficiency). Thus, one reason why focusing on the (less distant) club movement resulted in better learning than focusing on the (more distant) ball trajectory might be that it provided more salient information about the movement technique. Whether or not this is also true for expert performers remains open to question. It is possible that, for an automated skill, it does not matter whether the performer focuses on the outcome of the action-that is, a relatively distant effect-or on a closer effect that is more related to the technique. This issue needs to be addressed in future research.

\section{Summary}

Overall, the studies reviewed above provide consistent evidence that motor skill performance and learning can be enhanced by giving learners instructions that direct their attention to the effects of their movements. That is, inducing such an external focus of attention has been shown to be more effective than directing attention to the movements themselves (internal focus) or to some other external cue that will prevent learners from focusing on their movements (e.g., Singer, 1985, 1988). Furthermore, focusing on a more remote effect seems to facilitate the discriminability of the effect from the body movements that produced it and to be more beneficial than focusing on a very close effect. There might be an optimal distance of the effect, at which it is easily distinguishablefrom the body movements but at which it is also still possible for the performer to relate this effect to the movement technique.

These findings could also have implications for other aspects of training, such as the feedback given to learners attempting to acquire a new skill. The purpose of providing learners with feedback is to guide them to the optimal movement form (with the goal often being to maximize the movement outcome, such as the distance or accuracy of a thrown object) by informing them about their deviations from the goal movement. Often, such feedback is therefore directed at the performer's movement coordination. That is, it promotes an internal focus of attention, which-on the basis of the findings from studies concerned with the attentional focus induced by instructions - might not be optimal for learning. Studies that have examined the effectiveness of feedback as a function of the learner's attentional focus will be reviewed in the next section.

\section{FEEDBACK AND ATTENTIONAL FOCUS}

\section{Feedback Effectiveness as a Function of the Learner's Attentional Focus}

The first study to investigate whether the effectiveness of feedback depends on the focus of attention induced by it was done by Shea and Wulf (1999). An interesting feature of that study was that the feedback presented to learners was actually identical and only its interpretation was manipulated. Shea and Wulf used the stabilometer task and presented two groups with concurrent visual feedback, which essentially consisted of the platform movements being displayed on a computer screen. One group of learners was informed that the feedback represented their own movements (internal focus), whereas the other group was told that the feedback represented lines that were attached to the platform in front of each of the performer's feet (external focus). In addition, there were internal- and external-focus (control) groups that were not provided feedback but were instructed to focus on their feet or the lines, respectively.

The retention results showed that learning was more effective not only when performers were given externalfocus instructions, but also when they were provided with external-rather than internal-focus feedback. That is, even though the feedback display was identical for the two feedback groups, the group interpreting the feedback as external performed better than the group interpreting it as internal. This suggests that the effectiveness of feedback can also be enhanced if it directs the performer's attention 
to the movement effects, rather than to the movements themselves.

Interestingly, the on-line feedback provided to learners in the Shea and Wulf (1999) study was generally more effective than no feedback in enhancing the learners' ability to maintain their balance- even though it could be argued that the feedback was redundant to their intrinsic (visual and kinesthetic) feedback (e.g., Magill, Chamberlin, \& Hall, 1991). Shea and Wulf argued that this added benefit of feedback could be due to the fact that the feedback served to induce an external focus of attention, independent of the (internal- or external-focus) instructions given to learners. That is, the visual feedback display might have induced a more remote focus of attention (see The Influence of the Distance of the External Effect section, above). In addition, the display information might have provided a constant and powerful reminder to maintain an external focus.

Furthermore, the withdrawal of the feedback display in retention had no detrimental effect on performance. This is in contrast to other studies, where the withdrawal of concurrent feedback resulted in clear performance decrements (e.g., Schmidt \& Wulf, 1997; van der Linden, Cauraugh, \& Greene, 1993; Winstein et al., 1996). Apparently, if the feedback presented to the learner becomes incorporated into the movement representation, performance becomes dependent on the feedback and is disrupted when this feedback is withdrawn (Henry, 1968; Schmidt, 1991). This would be expected if the function of augmented feedback is mainly informational (Salmoni, Schmidt, \& Walter, 1984; Schmidt, 1991), as is probably the case in typical feedback studies, where other sources of visual feedback are "artificially" removed. However, if feedback also serves to direct the learner's focus of attention, as in the Shea and Wulf (1999) study, in which no additional information could be gained from the feedback, no such decrements should occur. The fact that performance was indeed maintained in the absence of feedback cannot be explained by the informational role of feedback. Rather, these findings-together with the performance-enhancing effects of this feedback, despite its redundancy with the performer's intrinsic feedbacksuggest that feedback can have the capacity to induce an external focus of attention that benefits performance and learning.

In contrast to the experimental technique used by Shea and Wulf (1999), where the feedback given to different groups of participants was identical, in practical settings coaches or instructors typically provide the learner with verbal feedback that refers to that aspect of performance that needs the most improvement. That is, on the basis of what the coach considers to be the critical mistake or flaw, he or she gives feedback that will hopefully help the performer to make appropriate changes on subsequent attempts. The goal of a study by Wulf, McConnel, Gärtner, and Schwarz (in press) was, therefore, to examine the generalizability of the external-focus feedback benefits to the learning of a sport skill-that is, the volleyball "tennis" serve-under conditions that approximate those of athletic training situations. For this purpose, different feedback statements were selected that are often used in volleyball training and that refer to the performer's body movements (internal-focus feedback). These statements were "translated" into statements that basically contained the same information but directed the learners' attention more to the movement effects. For example, instead of instructing learners to shift their weight from the back leg to the front leg while hitting the ball (internal focus), they were instructed to shift their weight toward the target (external focus). After every fifth practice trial, the performer was provided with the feedback statement that was deemed most appropriate on the basis of his or her performance on the previous trials.

The results showed that the accuracy of the serves was greatly enhanced by the external-focus, relative to the internal-focus, feedback not only during practice, but also after a 1-week retention interval in a retention test without feedback. That is, the feedback that avoided direct references to the performer's body movements led to a greater accuracy in hitting a target. This was true not only for novices, but also for advanced players who already had experience with the "tennis" serve. Furthermore, this advantage in the movement outcome was not accomplished at the expense of movement form. As determined through expert ratings, both types of feedback led to similar improvements in form. That is, although the movement form in the novice group was degraded by internal-focus feedback during practice, this group caught up with the external-focus group in the retention test, where the feedback was withdrawn. Apparently, these participants "recovered" from the detrimental effects of the feedback that directed their attention to their movement coordination in the no-feedback retention test. For the experts, externalfocus feedback tended to result in a better movement form than did internal-focus feedback during both practice and retention.

The results of a recent study by Todorov, Shadmehr, and Bizzi (1997) also suggest that feedback about the movement effect appears to be more beneficial than feedback related to the movements that produced it. Todorov et al. argued that the highest level of motor planning and control seems to be in terms of the kinematics of the endeffector and that, therefore, the feedback given to the learner should be most effective if it represents the movements of the end-effector, rather than the body movements. Even though they did not compare these two types of feedback, Todorov et al. showed that the learning of table tennis strokes was enhanced by providing performers with concurrent feedback about the trajectory of their paddle (in relation to the paddle trajectory of an expert). Those participants receiving this type of feedback were more accurate in hitting the target than were the participants who were provided verbal feedback (on gross errors) and who hit 50\% more balls. It is conceivable that at least part of the reason for the effectiveness of feedback about the paddle motion was that it induced an external focus of attention, 
whereas the control participants (without feedback) paid more attention to their own movement patterns.

\section{Theoretical Implications for \\ Feedback Procedures}

The findings by Shea and Wulf (1999) and Wulf, McConnel, et al. (in press) showing a differential effectiveness of feedback as a result of the focus of attention it induces have not only practical implications for the training of motor skills, but also theoretical implications. The current predominant view with respect to the function of feedback for motor learning holds that feedback manipulations are most effective if they enhance the learners' awareness of their body movements (e.g., Salmoni et al., 1984; Schmidt, 1991; Schmidt \& Lee, 1999). That is, it is assumed that conscious control of movements is essential for learning to be effective, especially early in the learning process (Adams, 1971; Fitts, 1964). The findings of numerous recent studies have been interpreted as providing evidence for the view that learning is made more effective by giving learners a chance to attend to their movements-for example, by providing feedback only on a portion of trials (e.g., Nicholson \& Schmidt, 1991; Winstein \& Schmidt, 1990; Wulf \& Schmidt, 1989; Wulf, Schmidt, \& Deubel, 1993), or by giving summary feedback (e.g., Schmidt, Lange, \& Young, 1990; Schmidt, Young, Swinnen, \& Shapiro, 1989) or average feedback (e.g., Wulf \& Schmidt, 1996; Young \& Schmidt, 1992).

These findings have been interpreted in terms of the guidance hypothesis (Salmoni et al., 1984; Schmidt, 1991), according to which feedback guides the learner to the correct response, reducing errors and facilitating performance. However, frequent (single-trial) feedback is also argued to have negative effects for learning, such as the learner's becoming too dependent on this informational support and the tendency for feedback to block the processing on intrinsic postresponse feedback. In other words, frequent feedback is assumed to prevent learners from focusing on their own movements, which is seen as a precondition for the development of an effective movement representation.

Given the fact that the feedback provided in most experiments seemed to have directed the participants' attention to their movements, the finding that such an internal focus is not beneficial for learning suggests a different interpretation of the detrimental effects of frequent feedback. Rather than preventing learners from focusing on their own movements, as is implied by the guidance hypothesis, it is possible that providing learners with frequent feedback actually makes them focus too much on their movements, leading to the typically observed learning decrements. Reducing the relative feedback frequency (or providing summary or average feedback), on the other hand, might give the learner a chance to perform the movement without being too concerned about his or her performance. Even though this does not necessarily induce an external focus of attention, not focusing on the move- ment itself still seems to be more effective than directing one's attention to one's own performance (Singer et al., 1993; Wulf \& Weigelt, 1997).

The results of a recent study by Weeks and Kordus (1998) seem to provide support for this interpretation. In their study, different groups of participants practicing the soccer throw-in were given knowledge of performance (KP) - similar to the internal-focus feedback given in the study by Wulf, McConnel, et al. (in press) — either after every trial $(100 \%)$ or after every third trial $(33 \%)$. The results showed learning advantages for the $33 \%-\mathrm{KP}$ group, relative to the $100 \%$-KP group. Interestingly, the benefits for the $33 \%$-KP condition were seen not only in retention and transfer tests without KP, but also during practice. The reduced-KP benefits are contrary to the predictions of the guidance hypothesis. These findings make sense from an attentional focus point of view, however, in that the $100 \%$-KP condition was detrimental to performance and learning because of the constant internal focus it induced. Overall, the findings regarding the influence of the attentional focus induced by the feedback given to the learner suggest that a revision of our views regarding the role of feedback for motor skill learning might be necessary.

\section{ATTENTIONAL FOCUS AND MOTOR CONTROL}

The differential effects of an external versus internal focus of attention on motor performance and learning raise the question of what the underlying reasons for this phenomenon are. McNevin et al. (2001; see also Wulf, McConnel, et al., in press) argued that when participants are asked to focus on effects (markers on the stabilometer platform) that are in close proximity to their bodyor, as in the case of internal focus of attention conditions, to actually focus on their limbs-they might be more likely to actively intervene in control processes than are participants who are asked to focus on more distant effects. By attempting to actively control the movements, such as those involved in balancing on the stabilometer, the participants may inadvertently disrupt relatively automatic processes that normally control the movement.

In McNevin et al.'s (2001) study, additional analyses indeed revealed that different attentional foci seem to be associated with different motor control processes, which presumably are responsible for the observed performance and learning differences. McNevin et al. (2001) determined the frequency characteristics (fast Fourier transformation; FFT $)^{2}$ of the balance records of participants balancing on the stabilometer. It has been shown that constrained or compromised perceptual-motor systems exhibit lower frequency components relative to unconstrained systems, whereas higher frequency components seem to be an indication of the incorporation and coordination of additional available degrees of freedom (see Thompson \& Stewart, 1986) - a characteristic associated with skilled performance. For example, FFT analy- 
ses of finger or hand tremor of motor systems that are compromised by disease or aging (e.g., Gantert, Honerkamp, \& Timmer, 1992; Newell, Gao, \& Sprague, 1995) or for balance when inputs (e.g., vision) into the vestibular-occular system have been perturbed (e.g., Gurfinkel, Ivanenko, Levik, \& Babakova, 1995) yield tremor and balance records characterized by larger amplitude and slower frequencies, relative to the normal conditions. When the system is not compromised, hand and finger tremor is almost imperceptible to the eye because the driving frequency is relatively high, which also results in relatively small amplitudes. In essence, higher frequency responding is seen as an exploitation of and integration of the available perceptual-motor degrees of freedom (see Newell \& Slifkin, 1996, for a discussion of this issue).

McNevin et al. (2001) therefore argued that, if it is the case that active intervention in control processes constrains or compromises the neurological degrees of freedom associated with maintaining balance, frequency components similar to those identified in constrained or compromised perceptual-motor systems would be expected. Their results indeed showed that the two groups that focused on the distant markers clearly made more and smaller corrections in maintaining their balance on the platform than the group that focused on the markers close to the feet. That is, the former groups exhibited higher frequency and lower amplitude movements than did the latter group. More recently, Wulf, Shea, and Park (in press) found even higher amplitude and lower frequency movement adjustments for participants who focused on their feet (internal focus), as compared with participants focusing on markers in front of their feet (external focus). Presumably, the former participants tried to exert conscious control to an even greater extent.

McNevin et al. (2001; see also Wulf et al., 2001) proposed a constrained action hypothesis to account for these results. According to this hypothesis, performers focusing on their body movement, or on an effect that occurs in close proximity to their body, tend to actively intervene in the maintenance of a stable posture more than do performers focusing on a more distant effect. Given that balance on an unstable surface must be maintained with small, very rapid (reflexive) patterns of activation, it appears reasonable to assume that active intervention in these relatively automated processes may degrade, rather than enhance, a coordinated effector output. Thus, one advantage of focusing attention on the movement effect seems to be that it allows unconscious processes to control the movements required to achieve this effect, resulting in fast movement adjustments and generally enhanced performance and learning.

\section{THEORETICAL ACCOUNTS The Ideomotor Principle and Common-Coding Theory}

The idea that actions are controlled more effectively if attention is directed to the (intended) outcome of the ac- tion, or its remote effects, rather than to the close effects that are directly associated with it (e.g., kinesthetic feedback), can be traced back to Lotze (1852) and James (1890). To illustrate the point that remote effects are often more important than the action itself (or its close effects), James used the example of a reaching movement: "Keep your eye at the place aimed at, and your hand will fetch [the target]; think of your hand, and you will likely miss your aim" (James, 1890, Vol. 2, p. 520). These ideas can be traced back to Lotze's work (Lotze, 1852). In his view, the execution of body movements is always tightly coupled to representations of their effects. This link can be used in either direction - that is, from execution to representation or from representation to execution. James summarized these ideas in his famous ideomotor principle of human action: "Every representation of a movement awakens in some degree the actual movement which is its object; and awakens it in a maximum degree whenever it is not kept from doing so by an antagonistic representation present simultaneously in the mind" (James, 1890, Vol. 2, p. 526).

How do movement representations acquire the power to induce the execution of the movements which they represent? Lotze (1852) and James (1890) both argue that there is no mystery at all. Rather, the impulsiveness of cognition derives from previous learning. When a motor act is performed (for whatever cause or reason), it goes along with a number of perceivable effects. Some are close to the action in the sense of being accompaniments of the act's execution (kinesthetic sensations, etc.). Others may be more remote, like the fact that a light goes on at a distance when one's fingers operate a light switch. Such regular links between motor acts and perceivable bodily and environmental events can be used and exploited in two ways. The first leads from actions to effects, as, for example, in predicting or expecting an ongoing action's consequences. The second goes the reverse direction and leads from effects to actions, as, for example, in selecting and initiating a certain act on the basis of an intention to achieve certain effects.

This latter relationship-which leads from intended effects to acts-is considered the functional basis of the ideomotor principle. Any representation of an event of which we know from previous learning that it either accompanies or follows from a particular action will hereafter have the power to call forth the action that produces the event. This will, in the first place, apply to any idea that refers to bodily movements themselves (e.g., thinking of one's finger operating a light switch); but in the second place, it will also apply to ideas that refer to more remote action effects (e.g., thinking of the light's going on).

More recently, Prinz and colleagues (Prinz, 1992, 1997; Prinz, Aschersleben, Hommel, \& Vogt, 1995) and Hommel and colleagues (Elsner \& Hommel, 2001; Hommel, 1996; Hommel \& Elsner, 2000) have taken up James's and Lotze's ideas that actions are planned and controlled by their intended effects (action effect hypothesis; Prinz, 1997; see Hommel, Müsseler, Aschersleben, \& Prinz, in press, for a recent overview). In their studies on learning 
contingencies between actions and their effects, Hommel and Elsner have shown that environmental events that are initially produced by certain actions (action effects) then and thereby acquire the capability of selecting and triggering those actions (Elsner \& Hommel, 2001; Hommel $\&$ Elsner, 2000). Furthermore, in his theory of common coding of perception and action, Prinz $(1990,1997)$ has provided a possible explanation for the advantages of focusing on the effects of one's movements, rather than on the movements themselves. In contrast to traditional views, which assume that there are different and incommensurate coding systems for afferent and efferent information (e.g., Massaro, 1990; Sanders, 1980; Welford, 1968), Prinz (1992) argues that there is a common representational medium for perception and action. According to the common-coding view, efferent and afferent codes can be generated and maintained in a commensurate way only at a distant level of representation. That is, perception and action planning both refer to distal events, since this is the only format that allows for commensurate coding and, thus, for the planning of actions in a format shared with perception. Therefore, actions should be more effective if they are planned in terms of their intended outcome, rather than in terms of the specific movement patterns.

Thus, the advantages of focusing on the movement effects, as compared with focusing on the movements themselves, are in line with the assumptions of common-coding theory. Yet, because the theory is relatively abstract, it does not specifically predict the differential learning effects of external versus internal attentional foci. Therefore, we would like to suggest two tentative principles that may account for the influence that this factor has on the effectiveness of the attentional focus. The critical question is the following: Given a sequence of movement effects that the performer could focus on-such as, for example, the club motion in a golf swing, the trajectory of the ball, the landing point of the ball, and the final position of the ballwhich of these effects should the performer focus on in order to optimize performance? The first principle is that the effect that the performer focuses on should be as remote as possible. The second principle, which appears to contradict the first principle, is that the effect should be related as closely as possible to the action that produced it.

The first principle is based on findings showing that learning is enhanced to the extent that the distance of the attentional focus is increased (McNevin et al., 2001; Park et al., 2000). In these studies, balance learning was facilitated when the markers on the stabilometer platform that participants focused on were placed at greater distances from the body. The greater remoteness of the external cues that the attention is directed to seems to facilitate the discriminability of the effect from the body movements. The second principle is supported by the findings of Wulf et al. (2000, Experiment 2), which demonstrated that focusing on the less remote effect (motion of the golf club) can be more effective than focusing on a more remote effect (trajectory of the golf ball). These findings can be explained by taking into account that very re- mote effects, such as the trajectory of a golf ball, cannot be directly related to the body movements that produced it. That is, there is no direct relationship between a given trajectory and a particular movement pattern, since the same trajectory could have been produced by different coordination patterns. This ambiguity would be expected to make the learning of the correct technique and the development of a stable movement pattern-which, after all, is the goal of motor learning-relatively difficult. This problem is reduced when attention is directed to a less remote effect, such as the movement of the golf club. Because the club motion is more directly related to the body movements, in both space and time, this effect can be associated more easily with the motor commands that caused the club motion. Obviously, if anything is to be learned, it is necessary that the movement effects and the motor commands that produced these effects can be associated. Thus, the most effective attentional focus would appear to be one that represents a compromise between the two principles outlined above, with an optimal focus being directed to an effect that is as remote as possible but can still be related to the movements that caused it. These ideas are still relatively tentative, and more research using comparable tasks is needed before any firm conclusions can be drawn, which might eventually allow us to make testable predictions.

\section{RELATED FINDINGS}

Indications that directing one's attention to the (anticipated) effects of one's movements might be the more "natural" way of controlling one's actions, as compared with focusing on the details of coordinating the various submovements leading to this effect, can also be found in other areas. For example, support for this view comes from studies related to functional variability in movement production, as well as purposeful activity in occupational therapy. These findings are reviewed next.

\section{Consistency in the Movement Outcome Owing to Functional Variability}

One line of support for the notion that motor skills are controlled by their effects comes from studies that demonstrate that the variability in the movement outcome is often smaller than the combined variability in the movement parameters contributing to this outcome. For example, Loosch (1995; see also Müller \& Loosch, 1999) showed that in dart throwing, the variability in where the dart hit the board was smaller than the theoretical variability calculated from the variability in the vertical release angle and the velocity. That is, there were negative correlations between these parameters, indicating that a smaller release angle was compensated for by a higher velocity of the dart and vice versa. This was especially true for expert dart players, but was also seen in novices. Thus, the functional variability between these parameters results in a reduced variability in the outcome, which suggests that the motor system (automatically) controls the various de- 
grees of freedom in a way that ensures that the desired effect is achieved. That is, the motor system seems to take advantage of its degrees of freedom to accomplish the planned effect.

Loosch $(1993,1997)$ reviewed a number of studies demonstrating this phenomenon with different tasks. For example, early studies by Voigt (1933), examining the variability in the velocity of the center of mass and its angle during takeoff in long jumpers, and by Klemm (1930), looking at the overall time and the times at partial distances in running, found that the variability in the final result was smaller than the variability in its components. Also, the regulation of step lengths in long jumping (Lee, Lishman, \& Thomson, 1982) or horse vaulting (Trillhose, 1995 ) seems to suggest that a key variable in understanding the function of variability for movement control is the planned outcome or effect (Loosch, 1997, p. 301). Interestingly, these findings also are in line with Bernstein's (1967) contention that it is the goal of the task and the anticipation of the desired outcome that serve as invariants in the regulation of movements.

It is conceivable that adopting an external focus of attention - that is, focusing on the movement effect-facilitates compensatory variability in various movement parameters to ensure that the effect is achieved, whereas focusing on the movements themselves interferes with such processes. At least, this is an interesting hypothesis, which might further explain the benefits of an external focus, and its viability should be examined in future studies.

\section{Purposeful Activity in Occupational Therapy Enhances the Relearning of Motor Skills}

The concept of purposeful activity is central to theory and practice in occupational therapy. Purposeful activity is often contrasted with "pure" exercise, where the patient's attention is directed to the movement, rather than to the object or task (Huss, 1981; King, 1978). Adding a purpose or functional relevance to a task has generally been found to enhance the (re-)acquisition of motor skills in occupational therapy, as compared with simulated activity or exercise with a focus on movement coordination (e.g., Lang, Nelson, \& Bush, 1992; Steinbeck, 1986; Wu, Trombly, \& Lin, 1994; Wu, Trombly, Lin, \& Tickle-Degnen, 1998; Yoder, Nelson, \& Smith, 1989). In addition, purposeful activity has been shown to facilitate transfer from a practicing limb to a nonpracticed limb (Rice, 1998). Having patients after a cerebrovascular accident chop a mushroom with a real chopper, for example, has been found to result in more efficient, direct, and smooth movements, as compared with using a simulated chopper (Wu et al., 1998). Similarly, Wu et al. (1994) demonstrated that participants asked to pick up a pencil from a pencil holder and prepare to write their names (material-based occupation) showed more effective motor performance than did participants only pretending to perform the same movement (imagerybased occupation) or participants who were asked to simply reach forward (exercise). Wu et al. (1994) speculated that this advantage might be due to the fact that partici- pants in the material-based occupation might have focused on the pencil, as opposed to focusing on the movement process. This interpretation is in line with King's (1978) and Huss's (1981) proposal that motor control is facilitated by placing attention on the goal, rather than on the movements themselves. According to King, directing attention to a task or an object allows subconscious centers to organize a movement. Gliner (1985) therefore points out that a motor learning theory is needed that includes "purposeful activity in that it would emphasize that actor's attention to a particular object rather than to the internal aspects of the act" (Gliner, 1985, p. 29).

Differences between imitative, or pantomime, movements and movements with real objects are currently being investigated from other perspectives as well. Weiss, Jeannerod, Paulignan, and Freund (2000) looked at the temporal structure (e.g., relative and absolute durations of various movement segments) when participants imitated or actually performed a daily activity with real objects (drinking from a bottle with a glass). However, no considerable differences were observed in various kinematic parameters. Although it is possible that unimpaired individuals performing overlearned activities indeed show similar movement kinematics with or without the use of real objects, perhaps more sensitive performance measures would show subtle differences in, for example, the fluency of the movements. Also, more complex movements, such as tying shoe laces, that are more challenging for unimpaired persons-as chopping a mushroom might be for a braininjured patient — could reveal differences between imitative movements and movements with real objects that are not obvious for simple skills. At least, anecdotal evidence suggests that this might be the case.

\section{CONCLUSIONS}

The findings regarding attentional focus effects on performance and learning reviewed here have important implications for both motor learning theory and practice. From a practical point of view, they suggest that there is a considerable potential for enhancing the effectiveness and the efficiency of training procedures in basically any area in which motor skills have to be acquired (e.g., sports, physical or occupational therapy, music). Typically, the instructions and feedback provided in training settings are intended to make the learner aware of his or her movement coordination-under the assumption that this is a necessary precondition for the development of the proper movement technique. Recent evidence that conscious awareness and conscious control processes are often no more effective (e.g., Singer et al., 1993; Wulf et al., 1998) or even less effective than no instructions at all (Wulf \& Weigelt, 1997) puts into question the value of such information, however. The findings reviewed in the present paper suggest that learning can be greatly enhanced if references to the performers' movements are avoided as much as possible and if their attention is instead directed to the desired movement effect. 
One might argue that a drawback of these studies is that formal manipulation checks were not conducted in these studies, to verify that participants actually adopted the attentional focus they were instructed to use. Therefore, future studies might want to use participant interviews to verify the deployment of the instructed attentional foci. Nevertheless, the consistent differences between groups with external-focus versus internal-focus instructions across experiments suggest that the instructions were effective in producing these differences.

Another issue that should be addressed in future studies is how directing the learner's attention to the movement effect affects the acquisition of the proper technique. For example, if attention is directed to the trajectory of a golf ball or tennis ball, as opposed to the actual movement pattern, how exactly does this influence movement form? So far, only two studies have used expert ratings to evaluate movement quality under external- versus internal-focus conditions (Maddox et al., 1999; Wulf et al., 2001). Both of these studies found no differences in movement form. However, detailed and objective measurements might reveal more subtle form differences as a fuction of attentional focus. Also, Young and Schmidt (1992), for example, demonstrated that feedback about certain aspects of the movement pattern can have beneficial effects on movement outcome over and above feedback about the outcome.

Furthermore, future research should examine to what extent the performer's attentional focus affects the accuracy (bias) and/or variability of his or her performance. The scoring system used in our previous experiments using two-dimensional aiming tasks (Maddox et al., 1999; Wulf et al., 1999; Wulf et al., 2000) did not capture these performance characteristics (see Reeve, Fischman, Christina, \& Cauraugh, 1994, for a discussion of this problem). The utilization of measures suggested by Hancock, Butler, and Fischman (1995) could provide further insights into which aspects of performance are influenced by the performer's attentional focus.

Overall, it appears that the differential effects of the attentional focus manipulations could have important implications for applied settings. Simply wording instructions or feedback in a way that induces an external rather than an internal focus of attention could result in savings in terms of the time, money, or other resources required for training. Obviously, this is a concern in many areas, such as costintensive sports or clinical rehabilitation (McNevin, Wulf, \& Carlson, 2000). Optimizing motor performance and learning in this way might even lead to a reduction in the number of injuries or fatalities in potentially dangerous situations (e.g., skiing, motorcycle riding, hang-gliding).

The demonstrated effects of the learner's focus of attention on motor learning also suggest that there might be a need to reevaluate some of the theoretical assumptions regarding the learning process. As was pointed out earlier, many views of motor learning (e.g., Adams, 1971; Fitts, 1964; Meinel \& Schnabel, 1976) assume that conscious information processing is required-at least in the early stages of learning - for the learner to develop an adequate representation of the skill. Only later in learning are automatic control processes assumed to play a role. Consequently, it has been argued (e.g., Salmoni et al., 1984; Schmidt, 1991) that feedback should be provided in a way that allows learners to consciously attend to their intrinsic feedback (in order to enable them to detect and correct errors themselves later). However, on the basis of the learning advantages of not focusing on one's movements, relative to focusing on the movement effect, and the evidence for constrained action as a result of an internal attentional focus (McNevin et al., 2001; Wulf, Shea, \& Park, in press), the notion that conscious awareness is critical for learning to take place appears to be questionable, at least.

Common-coding theory (Prinz, 1990, 1997) provides a possible explanation for the advantages of focusing on the effects of one's movements, rather than on the movements themselves. Because, according to this theory, perception and action require a common representational medium, efferent and afferent codes are stored in the form of distal events. From this point of view, it makes sense to assume that actions will be more effective if they are planned in terms of their intended outcome or effect, rather than in terms of the specific movement patterns. On the basis of the findings reviewed in this article, we have proposed two principles that can be seen as specifications within this theoretical framework. According to these principles, an optimal focus of attention should be as remote as possible but, at the same time, allow the learner to relate the effect to the associated movements - since it is this relationship that needs to be learned. Future conceptions of motor learning need to accommodate these new findings and account for the role of the learner's attentional focus and its influence on learning.

\section{REFERENCES}

AdAms, J. A. (1971). A closed-loop theory of motor learning. Journal of Motor Behavior, 3, 111-150.

BAUMEISTER, R. F. (1984). Choking under pressure: Self-consciousness and paradoxical effects of incentives on skillful performance. Journal of Personality \& Social Psychology, 46, 610-620.

BERNSTEIN, N. A. (1967). The co-ordination and regulation of movements. Oxford: Pergamon.

BLIss, C. B. (1892-1893). Investigations in reaction time and attention. Studies from the Yale Psychology Laboratory, 1, 1-55.

BodER, D. P. (1935). The influence of concomitant activity and fatigue upon certain forms of reciprocal hand movements and its fundamental components. Comparative Psychology Monographs, 11 (No. 4).

Elsner, B., \& Hommel, B. (2001). Effect anticipation and action control. Journal of Experimental Psychology: Human Perception \& Performance, 27, 229-240.

FITTS, P. M. (1964). Perceptual-motor skills learning. In A. W. Melton (Ed.), Categories of human learning (pp. 243-285). New York: Academic Press.

Gallwey, W. T. (1982). The inner game of tennis. New York: Bantam Books.

Gantert, C. J., Honerkamp, J., \& Timmer, J. (1992). Analyzing the dynamics of hand tremor time series. Biological Cybernetics, 66, 479-484.

Garfield, C. A., \& Bennett, H. A. (1985). Peak performance: Mental training techniques of the world's greatest athletes. Los Angeles: Tarcher. 
Gliner, J. A. (1985). Purposeful activity in motor learning theory: An event approach to motor skill acquisition. American Journal of $\mathrm{OC}_{\mathrm{c}}$ cupational Therapy, 39, 28-34.

Gurfinkel, V. S., Ivanenko, P. Y., Levik, S. Y., \& Babakova, I. A. (1995). Kinesthetic reference for human orthograde posture. Neuroscience, 68, 229-243.

Hancock, G. R., Butler, M. S., \& Fischman, M. G. (1995). On the problem of two-dimensional error scores: Measures and analyses of accuracy, bias, and consistency. Journal of Motor Behavior, 27, 241 250.

HenRy, F. M. (1968). Specificity vs. generality in learning motor skill. In R. C. Brown Jr. \& G. S. Kenyon (Eds.), Classical studies in physical activity (pp. 328-331). Englewood Cliffs, NJ: Prentice-Hall.

Hомmes, B. (1996). Toward an action-concept model of stimulusresponse compatibility. In B. Hommel \& W. Prinz (Eds.), Theoretical issues of stimulus-response compatibility (pp. 281-320). Amsterdam: North-Holland.

Hommel, B., \& ElSner, B. (2000). Action as stimulus control. In A. Schick, M. Meis, \& C. Reckhardt (Eds.), Contributions to psychological acoustics (pp. 403-424). University of Oldenburg, Germany: BIS.

Hommel, B., Müsseler, J., Aschersleben, G., \& Prinz, W. (in press). The theory of event coding (TEC): A framework for perception and action. Behavioral \& Brain Sciences.

Huss, A. J. (1981). From kinesiology to adaptation. American Journal of Occupational Therapy, 35, 574-580.

JAMES, W. (1890). The principles of psychology (Vol. 2). New York: Holt.

Jeka, J. J., Schöner, G., Dijkstra, T., Ribeiro, P., \& Lackner, J. R. (1997). Coupling of fingertip somatosensory information to head and body sway. Experimental Brain Research, 113, 475-483.

Kimble, G. A., \& Perlmuter, L. C. (1970). The problem of volition. Psychological Review, 77, 361-384.

KING, L. J. (1978). Toward a science of adaptive responses. American Journal of Occupational Therapy, 32, 429-437.

KLATZKy, R. L. (1984). Memory and awareness: An informationprocessing perspective. New York: Freeman.

KLemm, O. (1930). Gedanken über Leibesübungen [Thoughts on physical education]. Neue Psychologische Studien, 9, 145-167.

Lang, E. M., Nelson, D. L., \& Bush, M. A. (1992). Comparison of performance in materials-based occupation, imagery-based occupation, and rote exercise in nursing home residents. American Journal of Occupational Therapy, 46, 607-611.

Lee, D. N., Lishman, J. R., \& Thomson, J. A. (1982). Regulation of gait in long jumping. Journal of Experimental Psychology: Human Perception \& Performance, 8, 448-459.

Lidor, R. Tennant, L. K., \& Singer, R. N. (1996). The generalizability effect of three learning strategies across motor task performances. International Journal of Sport Psychology, 27, 23-36.

Loosch, E. (1993). Das Ganze läuft genauer ab als seine Teile [The whole runs more accurately than its parts]. Sportpsychologie, 7, 26-30.

Loosch, E. (1995). Funktionelle Variabilität in Dartwurf [Functional variability in dart throwing]. Sportwissenschaft, 25, 417-425.

Loosch, E. (1997). Variabilität: Phänomen und Prinzip menschlicher Bewegung [Variability: Phenomenon and principle of human movement]. Sportwissenschaft, 27, 294-309.

Lotze, R. H. (1852). Medicinische Psychologie oder Physiologie der Seele [Medical psychology or physiology of the soul]. Leipzig: Weidmann'sche Buchhandlung.

Maddox, M. D., Wulf, G., \& Wright, D. L. (1999). The effect of an internal vs. external focus of attention on the learning of a tennis stroke. Journal of Exercise Psychology, 21, S78.

Magill, R. A., Chamberlin, C. J., \& Hall, K. G. (1991). Verbal knowledge of results as redundant information for learning an anticipation timing skill. Human Movement Science, 10, 485-507.

Massaro, D. W. (1990). An information-processing analysis of perception and action. In O. Neumann \& W. Prinz (Eds.), Relationships between perception and action: Current approaches (pp. 133-166). Berlin: Springer-Verlag.

MASTERS, R. S. W. (1992). Knowledge, knerves and know-how: The role of explicit versus implicit knowledge in the breakdown of a complex motor skill under pressure. British Journal of Psychology, 83, 343-358.
McNevin, N. H., Shea, C. H., \& Wulf, G. (2001). Increasing the distance of an external focus of attention enhances learning. Manuscript submitted for publication.

McNevin, N. H., Wulf, G., \& Carlson, C. (2000). Effects of attentional focus, self-control, and dyad training effects on motor learning: Implications for physical rehabilitation. Physical Therapy, 80, 373 385 .

Meinel, K., \& Schnabel, G. (1976). Bewegungslehre [Movement science]. Berlin: Sportverlag.

Müller, H., \& LoOSCH, E. (1999). Functional variability and an equifinal path of movement during targeted throwing. Journal of Human Movement Studies, 36, 103-126.

Newell, K. M., GaO, F., \& Sprague, R. L. (1995). The dynamics of finger tremor in tardive dyskinesia. Chaos, 5, 43-47.

Newell, K. M., \& SLIFKIN, A. B. (1996). The nature of movement variability. In J. Piek (Ed.). Motor control and human skill: A multidisciplinary perspective (pp. 143-159). Champaign, IL: Human Kinetics.

Nicholson, D. E., \& SchmidT, R. A. (1991). Scheduling information feedback to enhance training effectiveness. In Proceedings of the Human Factors Society 35th Annual Meeting (pp. 1400-1403). Santa Monica, CA: Human Factors Society.

Park. J.-H., Shea, C. H., McNevin, N. H., \& Wulf, G. (2000, June). Attentional focus and the control of dynamic balance. Paper presented at the annual meeting of the North American Society for the Psychology of Sport and Physical Activity, San Diego.

Prinz, W. (1990). A common coding approach to perception and action. In O. Neumann \& W. Prinz (Eds.), Relationships between perception and action (pp. 167-201). Berlin: Springer-Verlag.

Prinz, W. (1992). Why don't we perceive our brain states? European Journal of Cognitive Psychology, 4, 1-20.

PrINZ, W. (1997). Perception and action planning. European Journal of Cognitive Psychology, 9, 129-154.

Prinz, W., Aschersleben, G., Hommel, B., \& Vogt, S. (1995). Handlungen als Ereignisse [Actions as events]. In D. Dörner \& E. van der Meer (Eds.), Das Gedächtnis: Probleme-Trends-Perspektiven (pp. 129-168). Göttingen: Hogrefe.

Reeve, T. G., Fischman, M. G., Christina, R. W., \& Cauraugh, J. H. (1994). Using one-dimensional task error measures to assess performance on two-dimensional tasks: Comment on "attentional control, distractors, and motor performance." Human Performance, 7, 315319.

RICE, M.S. (1998). Purposefulness and cross transfer in a forearm supination and pronation task. Scandinavian Journal of Occupational Therapy, 5, 31-37.

Riley, M. A., Stoffregen, T. A., Grocki, M. J., \& Turvey, M. T. (1999). Postural stabilization for the control of touching. Human Movement Science, 18, 795-817.

Salmoni, A. W., Schmidt, R. A., \& Walter, C. B. (1984). Knowledge of results and motor learning: A review and critical appraisal. Psychological Bulletin, 95, 355-386.

SANDERS, A. F. (1980). Stage analysis of reaction processes. In G. E. Stelmach \& J. Requin (Eds.), Tutorials in motor behavior (pp. 331-354). Amsterdam: North-Holland.

Schmidt, R. A. (1988). Motor control and learning: A behavioral emphasis (2nd ed.). Champaign, IL: Human Kinetics.

ScHMIDT, R A. (1991). Frequent augmented feedback can degrade learning: Evidence and interpretations. In J. Requin \& G. E. Stelmach (Eds.), Tutorials in motor neuroscience (pp. 59-75). Dordrecht: Kluwer.

Schmidt, R. A., Lange, C., \& Young, D. E. (1990). Optimizing summary knowledge of results for skill learning. Human Movement Science, 9, 325-348.

Schmidt, R. A., \& LeE, T. D. (1999). Motor control and learning: A behavioral emphasis (3rd ed.). Champaign, IL: Human Kinetics.

Schmidt, R. A., \& Wulf, G. (1997). Continuous concurrent feedback degrades skill learning: Implications for training and simulation. Human Factors, 39, 509-525.

Schmidt, R. A., Young, D. E., Swinnen, S., \& Shapiro, D. E. (1989). Summary knowledge of results for skill acquisition: Support for the guidance hypothesis. Journal of Experimental Psychology: Learning, Memory, \& Cognition, 15, 352-359.

SchNeIDER, W., \& Fisk, A. D. (1983). Attention theory and mechanisms 
for skilled performance. In R. A. Magill (Ed.), Memory and control of action (pp. 119-143). Amsterdam: North-Holland.

SheA, C. H., \& Wulf, G. (1999). Enhancing motor learning through external-focus instructions and feedback. Human Movement Science, 18, 553-571.

SingER, R. N. (1985). Sport performance: A five-step mental approach. Journal of Physical Education \& Recreation, 57, 82-84.

SINGER, R. N. (1988). Strategies and metastrategies in learning and performing self-paced athletic skills. Sport Psychologist, 2, 49-68.

Singer, R. N., Cauraugh, J. H., Tennant, L. K., Murphey, M., Chen, D., \& Lidor, R. (1991). Attention and distractors: Considerations for enhancing sport performances. International Journal of Sport Psychology, 22, 95-114.

Singer, R. N., Lidor, R., \& CAuraugh, J. H. (1993). To be aware or not aware: What to think about while learning and performing a motor skill. Sport Psychologist, 7, 19-30.

Singer, R. N., Lidor, R, \& Cauraugh, J. H. (1994). Focus of attention during motor skill performance. Journal of Sports Sciences, 12, 335-340.

Singer, R. N., \& Suwanthada, S. (1986). The generalizability effectiveness of a learning strategy on achievement in related closed motor skills. Research Quarterly for Exercise \& Sport, 57, 205-214.

Steinbeck, T. M. (1986). Purposeful activity and performance. American Journal of Occupational Therapy, 40, 529-534.

Thompson, J. M. T., \& Stewart, H. B. (1986). Nonlinear dynamics and chaos. New York: Wiley.

Todorov, E., Shadmehr, R. \& Bizzi, E. (1997). Augmented feedback presented in a virtual environment accelerates learning of a difficult motor task. Journal of Motor Behavior, 29, 147-158.

Trilllhose, A. (1995). Zu Aspekten der Schrittgestaltung im Anlauf und des Brettaufsatzsprunges bei turnerischen Stützsprüngen am Pferd [Aspects concerning the step regulation and jump on the board in horse vaulting]. Leistungssport, 25, 37-39.

van der Linden, D. W., Cauraugh, J. H., \& Greene, T. A. (1993). The effect of frequency of kinetic feedback on learning an isometric force production task in nondisabled subjects. Physical Therapy, 73, 79-87.

VoIgT, E. (1933). Über den Aufbau von Bewegungsgestalten [About the organization of movement "Gestalts"]. Neue Psychologische Studien, 12, 1-45.

WeEKs, D. L., \& Kordus, R. N. (1998). Relative frequency of knowledge of performance and motor skill learning. Research Quarterly for Exercise \& Sport, 69, 224-230.

Weiss, P. H., Jeannerod, M., Paulignan, Y., \& Freund, H.-J. (2000). Is the organization of goal-directed action modality specif ic? A common temporal structure. Neuropsychologia, 38, 1136-1147.

Welford, A. T. (1968). Fundamentals of skill. London: Methuen.

Winstein, C. J., Pohl, P. S., Cardinale, C., Green, A., Scholtz, L., \& WATERS, C. S. (1996). Learning a partial-weight-bearing skill: Effectiveness of two forms of feedback. Physical Therapy, 76, 985-993.

Winstein, C. J., \& Schmidt, R. A. (1990). Reduced frequency of knowledge of results enhances motor skill learning. Journal of Experimental Psychology: Learning, Memory, \& Cognition, 16, 677-691.

Wu, C., Trombly, C. A., \& Lin, K. (1994). The relationship between occupational form and occupational performance: A kinematic perspective. American Journal of Occupational Therapy, 48, 679-688.

Wu, C., Trombly, C. A., Lin, K., \& Tickle-Degnen, L. (1998). Effects of object affordances on reaching performance in persons with and without cerebrovascular accident. American Journal of Occupational Therapy, 52, 447-456.

Wulf, G., Höß, M., \& PRINZ, W. (1998). Instructions for motor learning: Differential effects of internal versus external focus of attention. Journal of Motor Behavior, 30, 169-179.

Wulf, G., Lauterbach, B., \& ToOle, T. (1999). Learning advantages of an external focus of attention in golf. Research Quarterly for Exercise \& Sport, 70, 120-126.

Wulf, G., McConnel, N., Gärtner, M., \& Schwarz, A. (in press). Feedback and attentional focus: Enhancing the learning of the volleyball serve through external-focus feedback. Journal of Motor Behavior.

Wulf, G., McNevin, N. H., Fuchs, T., Ritter, F., \& Toole, T. (2000). Attentional focus in complex motor skill learning. Research Quarterly for Exercise \& Sport, 71, 229-239.

Wulf, G., McNevin, N. H., \& Shea, C. H. (2001). The automaticity of complex motor skill learning as a function of attentional focus. Quarterly Journal of Experimental Psychology, 54A, 1143-1154.

Wulf, G., \& SchmidT, R. A. (1989). The learning of generalized motor programs: Reducing the relative frequency of knowledge of results enhances memory. Journal of Experimental Psychology: Learning, Memory, \& Cognition, 15, 748-757.

Wulf, G., \& Schmidt, R. A. (1996). Average KR degrades parameter learning. Journal of Motor Behavior, 28, 371-381.

Wulf, G., Schmidt, R. A., \& Deubel, H. (1993). Reduced feedback frequency enhances generalized motor program learning but not parameterization learning. Journal of Experimental Psychology: Learning, Memory, \& Cognition, 19, 1134-1550.

Wulf, G., Shea, C. H. \& PARK, J.-H. (in press). Attention in motor learning: Preferences for and advantages of an external focus. Research Quarterly for Exercise \& Sport.

WULF, G., \& WEIGELT, C. (1997). Instructions about physical principles in learning a complex motor skill: To tell or not to tell. Research Quarterly for Exercise \& Sport, 68, 362-367.

Yoder, R. M., Nelson, D. L., \& Smith, D. A. (1989). Added purpose versus rote exercise in female nursing home residents. American Journal of Occupational Therapy, 43, 581-586.

Young, D. E., \& Schmidt, R. A. (1992). Augmented kinematic feedback for motor learning. Journal of Motor Behavior, 24, 261-273.

\section{NOTES}

1. The addition of an attentional focus instruction might be conceived as making the task more difficult by creating a dual-task situation. However, the absence of a general dual-task decrement, relative to the control condition, argues against such a view.

2. The FFT analysis is a signal-processing procedure that translates a function in the time domain into a function in the frequency domain. In the McNevin et al. (2001) study, FFT analysis was used to decompose the balance records into sinusoids of different frequencies. The resulting Fourier coefficients represented the contribution of each sine and cosine function at each frequency. Once the frequency content of the balance records had been identified, it was possible to identify the average driving frequency underlying the control of balance.

(Manuscript received May 31, 2000; revision accepted for publication January 12, 2001.) 